\title{
Soft X-ray components in the hard state of accreting black holes
}

\author{
Caroline D’Angelo ${ }^{* a}$, Dimitrios Giannios ${ }^{a}$, Cornelis Dullemond $^{b}$, Hendrik Spruit $^{a}$ \\ ${ }^{a}$ Max-Planck-Institut fuer Astrophysik \\ Karl-Schwarzchild-Str. 1, 85741 Garching, Germany \\ ${ }^{b}$ Max-Planck-Institut für Astronomie \\ Könistuhl 17, 69177 Heidelberg, Germany \\ E-mail: dangelo@mpa-garching.mpg.de, giannios@mpa-garching.mpg.de, \\ dullemon@mpia-hd.mpg. de, henk@mpa-garching.mpg.de
}

\begin{abstract}
Recent observations of two black hole candidates (GX 339-4 and J1753.5-0127) in the low-hard state $\left(L_{X} / L_{\mathrm{Edd}} \simeq 0.003-0.05\right)$ suggest the presence of a cool accretion disk very close to the innermost stable orbit of the black hole. This runs counter to models of the low-hard state in which the cool disk is truncated at a much larger radius. We study the interaction between a moderately truncated disk and a hot inner flow. Ion-bombardment heats the surface of the disk in the overlap region between a two-temperature advection-dominated accretion flow and a standard accretion disk, producing a hot $\left(k T_{\mathrm{e}} \simeq 70 \mathrm{keV}\right)$ layer on the surface of the cool disk. The hard $\mathrm{X}$-ray flux from this layer heats the inner parts of the underlying cool disk, producing a soft X-ray excess. Together with interstellar absorption these effects mimic the thermal spectrum from a disk extending to the last stable orbit. The results show that soft excesses in the low-hard state are a natural feature of truncated disk models.
\end{abstract}

VII Microquasar Workshop: Microquasars and Beyond

September 1- 5, 2008

Foca, Izmir, Turkey

\footnotetext{
*Speaker.
} 


\section{Contents}

1. Introduction 2

2. Ion-Bombardment model 3

3. Producing a model spectrum 5

3.1 Disk emission 5

3.2 Comptonization 6

$\begin{array}{lll}3.3 & \text { Model output } & 7\end{array}$

$\begin{array}{lll}\text { 4. Comparison to observations: SWIFT J1753.5-0127 } & 7\end{array}$

$\begin{array}{ll}\text { 5. Conclusions } & 9\end{array}$

\section{Introduction}

The geometry of the low-luminosity ("low-hard") state of Galactic Black Hole Candidates (GBHC), in which the spectrum is dominated by a power law X-ray flux extending to high energies, has been an open question for several decades. While it is generally believed that the power law spectrum is formed by inverse Compton scattering, there is no consensus about the geometry of the flow, source of seed photons or energy distribution for the Comptonizing electrons.

Broadly speaking, there are two classes of model to explain the spectrum in the low-hard state. The first is the "corona" model, in which the disk remains untruncated or nearly untruncated at luminosities $L_{\mathrm{X}} \simeq 10^{-3} L_{\mathrm{Edd}}$. The hard power law spectrum comes from a hot, patchy corona (perhaps powered by magnetic flares, e.g. (1)) on top of the disk, while the surrounding region is bombarded with high energy photons, producing the observed reflection and Fe-K fluorescence. In the alternate, "truncated disk" model the thin disk is truncated at some distance from the black hole and the inner region is filled with a hot, radiatively-inefficient flow, which produces the hard spectrum. The reflection spectrum and Fe-K fluorescence is then produced by the interaction of the hard X-rays with the inner part of the truncated disk, or in some cool outflow moving away from the disk. For a recent discussion of the low-hard state see sect. 4 of (2).

The presence or absence of a cool disk should be confirmable through direct detection of a soft X-ray blackbody component at low energies. However, this is made difficult by the fact that at low accretion rates the temperature of even an untruncated disk will drop from about $1-2 \mathrm{keV}$ in the high soft state to $\sim 0.1-0.3 \mathrm{keV}$, which puts it out of the range of most X-ray detectors. Additionally, the effects of interstellar absorption become very strong at around $0.1 \mathrm{keV}$, so that detecting a soft excess and accurately measuring its parameters will depend on how accurately the interstellar absorption can be determined. 
This question was the focus of two recent papers, $(3 ; 4)$, in which the authors studied longexposure XMM-Newton spectra of two different GBHCs, SWIFT J1753.5-0127 and GX 339-4, at low luminosities $\left(L_{\mathrm{X}} / L_{\mathrm{Edd}} \sim 0.003-0.05\right)$ to try to detect the disk directly. In both papers the authors report the detection of a very weak soft excess, which, when fit to blackbody models for a standard accreting disk give disks with a maximum temperature of $k T \sim 0.2-0.4 \mathrm{keV}$, and inner radius consistent with the innermost stable circular orbit of a black hole.

Determining the inner radius of an accretion disk from its spectrum relies on an accurate understanding of how luminosity in the disk is produced. When the (approximately) blackbody flux from the disk dominates the spectrum, the disk's inner radius can be inferred by assuming a known 'colour correction' for the blackbody spectrum, and that the radiation is produced by internal viscous dissipation in the disk as it accretes, provided the distance and inclination of the source are known.

However, when the spectrum becomes dominated by very hard X-ray and $\gamma$-ray radiation, the radial temperature and luminosity profile of the disk will depend on the interaction between the hard radiation and the disk. This is because most of the accretion power is now in the hot gas producing the hard radiation, some of which will interact with the cool disk (seen in reflection component and $\mathrm{Fe}-\mathrm{K}$ emission line), and may provide a substantial source of heating. This heating of the disk surface converts the hard radiation into a soft component. In the simplest version of this model, Haardt and Maraschi (5) calculated the energy balance for a hot corona covering a cool disk and found that the flux in the soft and hard components will be roughly equal. This predicts that a substantial soft X-ray component is a universal feature of the hard-state spectrum, whether the disk is truncated or not. Its detectability depends on the sensitivity of detectors in the $0.1-1 \mathrm{keV}$ range, and the interstellar absorption column.

For models in which a cool, truncated accretion disk encircles a very hot inner flow (which cools through inverse Compton scattering), there will necessarily be a region of interaction near the truncation radius, which will heat the inner edge of the disk. This will come either from hard photons bombarding the disk as in the Haardt-Maraschi model, or through matter interaction, with hot protons from the inner flow directly colliding with the cool disk. In both cases previous work has shown that a soft component is produced, but a more detailed model is needed to predict the radiation spectrum. The first case, examining the structure of a thin accretion disk bombarded by hard photons has been studied by for AGN disks (6) and (7), and more recently for GBHC disks by (8). In this talk we focus on the second case, in which a moderately truncated disk is embedded in a hot, two-temperature advection-dominated accretion flow (ADAF). Our goal is to determine whether such a model could reproduce the soft spectral components reported by $(3 ; 4)$. We will find that disks truncated at 15-20 Schwarzschild radii $\left(R_{\mathrm{S}} \equiv G M / \mathrm{c}^{2}\right)$ can in fact produce soft components of the observed strength and shape, which is demostrated with a qualitative comparison to the soft excess in (3). The work presented here is described in more detail in (9).

\section{Ion-Bombardment model}

We begin with the results from a prototype model for ion bombardment on cool disks, initially proposed by (10), and extended in (11), and (12) (hereafter DS05). In this model a cool disk is embedded in a two-temperature ADAF in which the protons are close to their virial temperature 

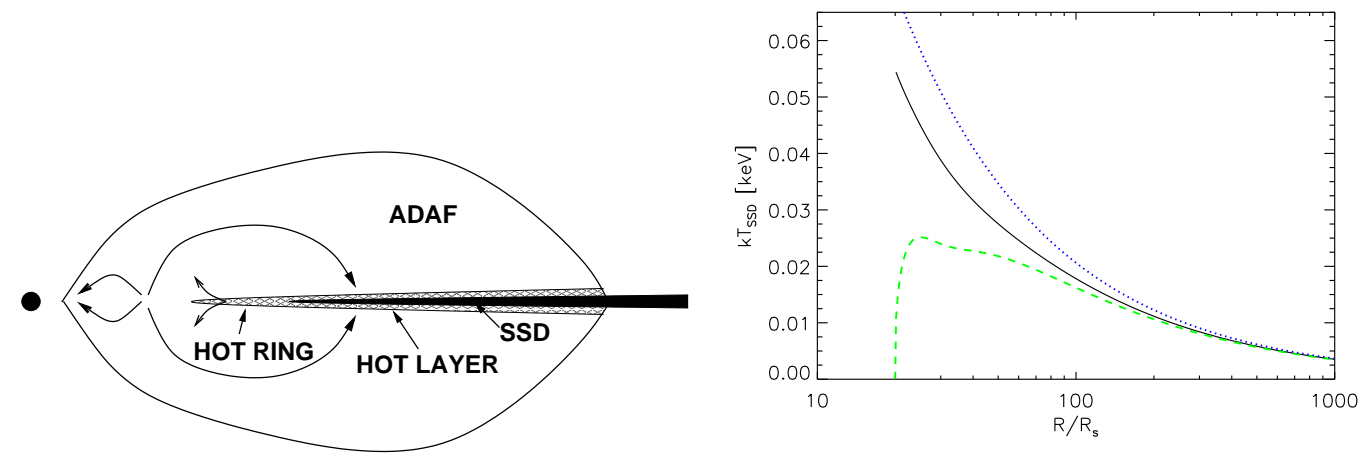

Figure 1: LEFT: Schematic structure of a cool disk embedded in an ADAF. Here the ADAF (transparent outer region) extends over the inner edge of the cool Shakura-Sunyaev disk (SSD), shown by the solid thin disk, bombarding it with energetic protons $\left(k T_{\mathrm{p}} \sim 20 \mathrm{MeV}\right)$. This evaporates the surface of the disk into a corona $\left(k T_{\mathrm{e}} \sim 70 \mathrm{keV}\right)$ (HOT LAYER, hatched). The higher viscosity of the hot layer causes it to spill over the inner edge of the SSD where cooling through inverse Compton scattering is no longer efficient (HOT RING) and thermal instability causes the layer to evaporate into the ADAF. The mass transfer is represented by the small arrows.

RIGHT: Effect of ion-bombardment on the surface temperature of a cool (SSD) disk truncated at 20RS. Green dashed line: radial temperature profile in disk without considering heating from hot layer. Black solid line: radial temperature profile when disk heating is considered. Blue dotted line: radial temperature profile for an untruncated disk at the same accretion rate.

$(\sim 20 \mathrm{MeV})$. The protons bombard the disk, and are stopped via Coulomb collisions with the disk's electrons, thus transferring their energy to the disk. The energy from the protons is sufficient to evaporate the upper layers of the disk into a hot corona (called here the "hot layer"), whose temperature is set by a balance between heating from the ions and cooling, predominantly through inverse Compton scattering of disk photons. The work in (13) found that the optical depth of the hot layer is around unity, varying only weakly with irradiating flux and distance from the hole.

The higher viscosity of the hot layer allows it to spill over inside the inner edge of the cool disk (see left panel of fig. 1), where the lack of seed photons (and hence inefficient cooling) in this layer will cause the temperature to rise to about $k T_{e} \sim 200-300 \mathrm{keV}$, and evaporate into the ADAF (11). This shows how an ion-supported flow can be maintained inside a truncated cool disk. The key for the whole process is the presence of a component of intermediate temperature (the hot layer). On one hand this component produces a hard, Comptonized spectrum, while on the other its evaporation feeds the ion-supported flow.

DS05 extended this work to examine the radial dependence of the three components of the flow. They set up a model in which the truncation radius of the cool disk is a free parameter, and the three layers are assumed to interact only via the mass transfer processes outlined above. They assumed the cool disk was a standard Shakura-Sunyaev disk (14), and that the ADAF flow temperature followed the model of Narayan and Yi (15). They approximated the temperature in the hot layer to be constant, and also assumed a constant temperature for its extension inside the inner edge of the cool disk (here called the "hot ring"). With these assumptions, the radial profiles of the three components can then be determined by solving the equations for mass and angular 
momentum conservation for a thin disk.

This simplified analysis yields estimates of the mass accretion rate $\dot{M}_{i}$, heating rate $Q_{i}$ (from internal viscous dissipation and ion heating) and surface density $\Sigma_{i}$ as a function of radius for each component. For further discussion of the model see section 2 of DS05. The input parameters for the model are the black hole mass $M_{B H}$, the magnitude of the $\alpha$ viscosity parameter (14) and the truncation radius $R_{S S D}$ for the disk. A sketch of the model with its various components is seen in the left panel of figure 1. Assuming the dominant radiation mechanisms are known, we can determine the spectrum from the resulting energy distribution for each layer. This is the primary goal of the current work.

\section{Producing a model spectrum}

The three components of the model exchange mass and energy. In the steady-state assumed here, the sources and sinks balance in each. The hot layer is heated in similar measure by viscous dissipation and the hot ions it absorbs from the ion flow above. It is cooled by Compton upscattering of disk photons passing through it, which will produce a hard X-ray spectrum.

The cool disk is heated in three ways: by internal viscous dissipation, by reprocessing of hard (Comptonized) X-rays from the hot layer above, and by the small number of hot ions from the ADAF that pass through the hot layer. The reprocessing of the hard X-rays is further divided into a fraction $a$ that is reflected and a fraction $(1-a)$ that is absorbed and assumed to thermalize completely. The surface temperature of the cool disk, assumed to be close to its effective temperature, is thus given by:

$$
\sigma_{\mathrm{B}} T_{e f f}^{4}=Q_{\mathrm{c}, \mathrm{visc}}+(1-a) F_{\mathrm{h}}^{-}+f_{\mathrm{c}} Q_{\mathrm{i}},
$$

where $Q_{\mathrm{c} \text {,visc }}$ is the viscous heating rate of the cool disk, $a$ its $\mathrm{X}$-ray reflection albedo, $F_{\mathrm{h}}^{-}$the flux of downward directed X-ray photons from the hot layer, and $f_{\mathrm{c}} Q_{\mathrm{i}}$ the fraction of energy flux in hot ions absorbed by the cool disk. The albedo $a$ of the disk will depend on its ionization structure, which we estimate in the next section.

To illustrate the model we first discuss the results for a reference model with representative parameters, then adjust the parameters to compare the model to observation in section 4 . We take $M_{\mathrm{BH}}=10 M_{\odot}$ for the mass $R_{\mathrm{in}}=20 R_{\mathrm{S}}$ for the truncation radius, and use $\alpha=0.2$ for all accretion components.

\subsection{Disk emission}

The emission from the disk will come from two components: the blackbody flux from the disk itself and the reflection component from the incident Comptonized radiation. To estimate the relative contribution for each we need to estimate the albedo for the disk, which can be derived from the ionization parameter $\xi \equiv 4 \pi F_{\mathrm{X}} n_{\mathrm{H}}^{-1}$, the ratio of the incident flux on the disk to the hydrogen number density in the disk. The higher the ionization in the upper layers of the disk, the more flux will be reflected without reprocessing.

Since the disk is stratified, we need to calculate the hydrogen number density in the region where most of the reprocessing takes place, which we assume will be around the electron scattering photosphere of the cool disk. Assuming hydrostatic balance in the disk the pressure at this depth 
is approximately $P \simeq g / k_{\mathrm{es}}$, where $g$ is the vertical component of the acceleration due to gravity at height $\tau_{\text {es }}=1$ above the midplane and $k_{\text {es }}$ is the electron scattering opacity . Assuming this height is about twice the nominal disk thickness $H=c_{\mathrm{s}} / \Omega_{\mathrm{K}}$, the pressure is $P \simeq 2 c_{\mathrm{s}} \Omega_{\mathrm{K}}$. Here $c_{\mathrm{s}}$ is the sound speed at the midplane of the disk, determined from the heating rate $Q_{v i s c}$ by a standard thin disk model. To fix the density corresponding to this pressure we assume the temperature in this region is the effective surface temperature that follows from the viscous heating rate. For the reference model, this gives an ionization parameter of $\xi \simeq 10 \mathrm{erg} \mathrm{cm} \mathrm{s}^{-1}$, which corresponds to an albedo of $a \simeq 0.2$. We use the XSPEC reflection model "REFLION" (16) with the given ionization parameter to approximate the reflection spectrum.

We assume that the rest of the incident radiation is completely reprocessed and thermalized by the disk, resulting in the modified temperature profile for the disk shown in the right panel of figure 1. The green dashed line shows the temperature profile considering only viscous dissipation, and assuming no coupling between the inner edge of the disk and the hot ring. The solid line shows the effect when heating from the hot layer is included. As can be seen in the figure, the effect of heating extends to large radii. The dotted line shows the temperature profile for a standard untruncated disk for the same accretion rate.

However, the disk's spectrum deviates from a simple multi-temperature blackbody because the flux emerging from the photosphere of the disk will be reduced chiefly by Thomson scattering in the upper layers of the disk. This results in a modified blackbody, which is often described by a "colour correction factor" $f_{\text {col }}$ measuring the effect as the ratio of colour temperature to effective temperature $T_{\mathrm{col}}=f_{\mathrm{col}} T_{\mathrm{eff}}$. The value for $f_{\mathrm{col}}$ will change the determination of the inner disk radius, since the luminosity will be depressed by a factor of $f_{\text {col }}^{-4}$ relative to the temperature, so that when colour correction is considered, the inner radius of the accretion disk will be increased by a factor $f_{\text {col }}$. In this work we have the added complication of the hot surface layer, which will heat the upper layers of the disk substantially and may change the spectral hardening. A detailed radiative transfer calculation for the surface distribution in the disk is beyond our scope however, so we assume $f_{\text {col }}=1.7(17)$, and note that the real value may be higher. The total disk spectrum of modified blackbody+reflection is now used as input for the Comptonization simulation.

\subsection{Comptonization}

To calculate the spectrum from inverse Compton scattering through the hot layer and hot inner ring we use a one-dimensional Monte Carlo simulation. The simulation assumes a slab geometry, with the cool optically thick disk below a much hotter surface layer with moderate optical depth. We use the disk emission from the previous section as seed photons and use a simulation with high enough resolution to capture emission lines in the spectrum.

The input parameters of the Comptonization calculation are the soft input spectrum, and the optical depth and temperature of the region. We can estimate the optical depth from the surface density in the hot layer (from the output of the DS05), and find the temperature iteratively so that the total energy flux in Comptonized photons matches the energy input into the hot layer.

The Comptonization from hot inner ring is similarly determined, except that its contribution is uncertain because the geometry is very different from a plane-parallel configuration. In particular, the contribution from seed photons from the disk is uncertain, which in turn will set the temperature of the region (since it will set the rate of cooling). We thus introduce a parameter $\zeta$, which 
represents the fraction of seed photons from the cool disk that cool the hot layer, (so that $1-\zeta$ goes to cool the hot ring) and adjust it to match the observed hardness of the spectrum. For the reference model we use the output of DS05 to set $\zeta=0.99$.

\subsection{Model output}

The output spectrum for the reference model is shown in the top-left panel of figure 2. The flow model of DS05 gives an accretion rate of $\dot{M}=2.2 \times 10^{-3} \dot{M}_{\text {Edd }}$ (assuming an efficiency of $10 \%$ ), and a luminosity $L=4.2 \times 10^{-4} L_{\text {Edd }}$. The total spectrum is shown in black, and the individual components are identified in the caption. The bottom panel shows the spectrum divided by a powerlaw with photon index $\Gamma=1.91$. There is small excess below $0.5 \mathrm{keV}$, even though the maximum disk temperature is only $0.05 \mathrm{keV}$. Except for the very low accretion rate and steepness in the power-law, we find a spectrum qualitatively similar to those of (3) and (4).

The luminosity of our reference model, of the order $10^{-4} L_{\mathrm{Edd}}$, is substantially lower than typically inferred for the Low-Hard state $\left(10^{-2}-10^{-3} L_{\mathrm{Edd}}\right)$. This is a consequence of the flow model in DS05 that forms the basis of our analysis. In it, the surface density of the hot layer is governed by the physics of the Coulomb interaction of the hot ions penetrating through it, and its temperature by the energy balance between it and the underlying disk. With temperature and surface density constrained in this way, the mass flux then depends only on the radial drift speed, i.e. the viscosity parameter. The actual mass flux is low because the temperature of the layer is only about $80 \mathrm{keV}$. This suggests that the current model is incomplete. In this work, however, we solve this problem by introducing a parameter $C$, by which the accretion rate (or equivalently the energy output of each component of the flow) is increased. For a more extensive discussion of the uncertainties of the model and possible solutions see (9).

With the introduction of the parameters $C$ and $\zeta$ (introduced in section 3.2) our model loses its predictive power, but the goal of this paper is to present a model that is plausible rather than precise in its details. In the next section we compare our spectra to the observation of SWIFT J1753.50127, and show that for reasonable values of accretion rate and temperature in the hot layer and inner ring, we can reproduce the observed soft excesses using a significantly truncated accretion disk.

\section{Comparison to observations: SWIFT J1753.5-0127}

To show how our model compares with the soft excesses we consider the spectrum from the source SWIFT J1753.5-0127. Miller et al. (3) took a $42 \mathrm{ksec} X M M-N e w t o n$ observation and estimated an X-ray luminosity $(0.5-10 \mathrm{keV})$ of $L_{\mathrm{X}} / L_{\mathrm{Edd}}=2.6 \times 10^{-3}(d / 8.5 \mathrm{kpc})^{2}(\mathrm{M} / 10 \mathrm{M} \odot)$. They fit the spectrum to a power law with a photon index $\Gamma=1.67(2-10 \mathrm{keV})$, and interstellar absorption of $N_{\mathrm{H}}=2.3 \times 10^{21} \mathrm{~cm}^{-2}$. Fitting the spectrum with an absorbed power law component alone reveals a small soft excess below $2 \mathrm{keV}$, which they fit to a disk with $k T_{\text {in }} \simeq 0.22 \mathrm{keV}$ and $\mathrm{R}_{\text {in }} \simeq \mathrm{R}_{\mathrm{S}}$ $\left(\mathrm{M} / 10 \mathrm{M}_{\odot}\right)(d / 8.5 \mathrm{kpc}) / \cos ^{1 / 2} i$.

A very truncated disk will be too cool to be observable in X-rays, while an untruncated disk will have a higher temperature than is observed (because of the heating from the corona). To compare with observed spectra we assume a moderate truncation radius of $15 R_{\mathrm{S}}$, which is qualitatively very different from an untruncated disk. We make the same assumptions as in (3) for mass $(\mathrm{M}=$ 

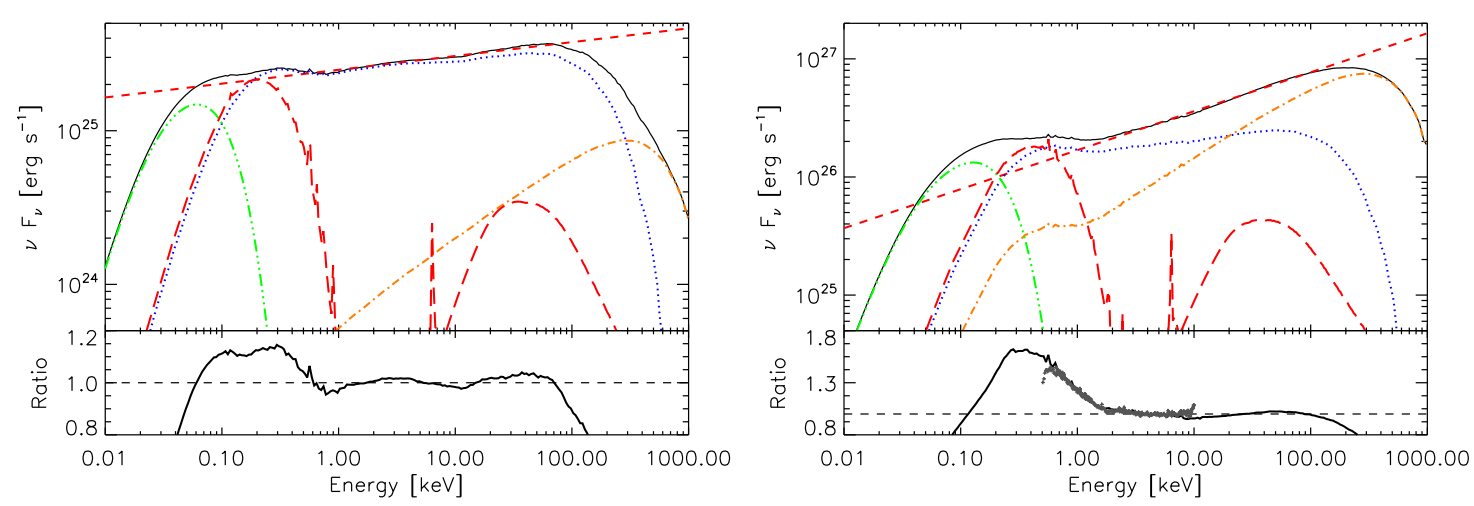

Figure 2: LEFT: Top: Relative contribution from each component for reference model, $\alpha=0.2, \mathrm{R}_{\text {in }}=20 \mathrm{R}_{\mathrm{S}}$, $\mathrm{M}=10 \mathrm{M}_{\odot}$. Red long-dashed line: Spectrum from the disk (modified blackbody plus reflection spectrum). Green dash-double dotted line: Spectrum from outer disk. Blue dotted line: Comptonized spectrum from hot layer. Orange dash-dotted line: Comptonized spectrum from hot ring. Black solid line: total spectrum. Red short-dashed line: power-law with $\Gamma=1.9$. Bottom: total spectrum divided by power-law with $\Gamma=1.9$ to show soft excess.

RIGHT: Top: Model spectrum for SWIFT J1753.5-0127, for a truncated disk with inner radius of $15 \mathrm{R}_{\mathrm{S}}, \alpha$ $=0.2$ and $\mathrm{M}=10 \mathrm{M}_{\odot}$. Components as in previous figure. Bottom: Soft excess in SWIFT J1753.5-1027. The crosses show the observed spectrum from (3) divided by an absorbed power-law with $\Gamma=1.66$ and interstellar absorption column $N_{\mathrm{H}}=2.3 \times 10^{21} \mathrm{~cm}^{-2}$. The solid line shows the ratio between our model ( $T o p$ ) and the same power-law, with $N_{\mathrm{H}}=2.35 \times 10^{21} \mathrm{~cm}^{-2}$.

$\left.10 \mathrm{M}_{\odot}\right)$ and distance $(d=8.5 \mathrm{kpc})$, and assume an inclination $\cos i=0.5$. To match the measured flux in the power-law component, we increase the flux in each component by a factor $C=12$, to give an accretion rate $\dot{M} / \dot{M}_{\text {Edd }}=1.7 \times 10^{-2}$ for an efficiency of $10 \%$.

In the hot layer, the predicted surface density profile gives an optical depth $\langle\tau\rangle=0.87$, and the energy balance between the cool disk and hot layer determines a temperature of $k T_{\mathrm{e}}=75 \mathrm{keV}$ for the hot layer, which gives a photon index of $\Gamma=1.87(2-10 \mathrm{keV})$. The density of the disk and flux in the hot layer also allows us to estimate the ionization parameter, $\xi=50 \mathrm{erg} \mathrm{cm} \mathrm{s}^{-1}$, from which we get an albedo and find the Comptonized flux incident on the disk heats it to $k T=0.11 \mathrm{keV}$, which looks like $k T=0.19 \mathrm{keV}$ when spectral hardening is taken into account.

The predicted surface density in the hot inner ring also allows us to calculate the optical depth in this layer, which we find to be $\langle\tau\rangle=0.7$. Since we do not have a detailed model for the radiative transfer in this component, we take a plausible value of $k T_{\mathrm{e}}=200 \mathrm{keV}$, which gives a photon index of $\Gamma=1.39$, and set $\zeta=0.89$ (the fraction of disk photons seeding the hot layer) to match the observed hardness in the spectrum.

The top-right panel of figure 2 shows the resultant spectral energy distribution for SWIFT J1753.5-0127. The different components shown in the figure are the same as in the left panel of figure 2. The bottom-right panel of figure 2 shows the observed spectrum divided by an absorbed power-law with an absorption column density with $N_{\mathrm{H}}=2.3 \times 10^{21} \mathrm{~cm}^{-2}$ (taken from (3)). Overlaid in black is the soft excess predicted by our model, where we have changed the absorption column density to $N_{\mathrm{H}}=2.35 \times 10^{21} \mathrm{~cm}^{-2}$. Very small changes in the estimated absorption column density and small deviations from a single power-law in the Comptonization spectrum (from consider- 
ing two regions of Comptonization as well as a plane-parallel geometry for the inverse Compton scattering) renders the two excesses effectively indistinguishable, even though the temperature and peak fluxes of both disks are very different. The bottom-right panel in figure 2 also shows that the deviation from a simple power-law in the range $2-100 \mathrm{keV}$ is less than $10 \%$, which is consistent with observation.

\section{Conclusions}

Our model of a disk truncated at $15-20 \mathrm{R}_{\mathrm{S}}$ and surrounding corona is qualitatively very different from the untruncated disk (with $R_{\text {in }}<3 R_{\mathrm{S}}$ ) models fit by $(3 ; 4)$, and it is natural to ask how the observed soft excess can be so small when the radiating area is so much larger. The answer lies in several points. The most important of these is that by accounting for colour correction, the temperature in our disks is about half that found by (3), so that the flux is intrinsically much smaller and (even after the colour correction is applied) most of the flux is cut off by interstellar absorption. There is a further reduction from the hot surface layer, which upscatters about two-thirds of the input photons. Finally, the shape of the upscattered photons deviates from a power-law at low energies, so that measuring the temperature of the soft excess depends very sensitively on modelling the Comptonized spectrum correctly.

From energetic considerations, the hard spectra observed in the low-hard state of LMXBs must be produced by hot $\left(k T_{\mathrm{e}} \sim 100 \mathrm{keV}\right)$ matter in the inner regions surrounding the black hole. If there is also a much cooler disk present, there will necessarily be some degree of interaction between the two components, and the disk will be heated by irradiation from the hot Comptonizing component. The fits reported in $(4 ; 3)$ neglect this interaction by fitting the disk and hard components separately. Here we have shown that incorporating the effects of this interaction heats the inner regions of a moderately truncated disk so that, when coupled with the effects of interstellar absorption, the size of the soft excess matches observations. Our work also highlights the potential pitfalls of using simple power-law or analytic Comptonization fits at low energies, which can provide significant deviations in the soft X-rays, thus changing the shape and intensity of the observed soft excess.

The model we have outlined provides several opportunities for further improvement in order to better constrain the introduced fitting parameters, $C$ and $\zeta$. The spectrum from the hot ring and ADAF are particularly uncertain, and dependent on a more detailed model for the radiative transfer through this region, as well as the source and number of seed photons (which will set the electron temperature in both regions). The model's global accretion rate (limited by the rate at which the hot layer spills over into the hot ring and then evaporates into the ADAF) is also very low, although this can be increased if the viscosity in the warm layer is increased, perhaps as a result of accretion through an ordered magnetic field.

\section{References}

[1] A. Merloni and A. C. Fabian, Accretion disc coronae as magnetic reservoirs, MNRAS 321 (Mar., 2001) 549-552, [arXiv: astro-ph/0009498]. 
[2] C. Done, M. Gierliński, and A. Kubota, Modelling the behaviour of accretion flows in X-ray binaries. Everything you always wanted to know about accretion but were afraid to ask, AEA Rev. 15 (Dec., 2007) 1-66, [arXiv:0708 . 0148].

[3] J. M. Miller, J. Homan, and G. Miniutti, A Prominent Accretion Disk in the Low-Hard State of the Black Hole Candidate SWIFT J1753.5-0127, ApJ 652 (Dec., 2006) L113-L116, [astro-ph/0605190].

[4] J. M. Miller, J. Homan, D. Steeghs, M. Rupen, R. W. Hunstead, R. Wijnands, P. A. Charles, and A. C. Fabian, A Long, Hard Look at the Low/Hard State in Accreting Black Holes, ApJ 653 (Dec., 2006) 525-535, [astro-ph/0602633].

[5] F. Haardt and L. Maraschi, A two-phase model for the X-ray emission from Seyfert galaxies, ApJ 380 (Oct., 1991) L51-L54.

[6] D. R. Ballantyne, R. R. Ross, and A. C. Fabian, X-ray reflection by photoionized accretion discs, MNRAS 327 (Oct., 2001) 10-22, [arXiv: astro-ph/0102040].

[7] S. Nayakshin and T. R. Kallman, Accretion Disk Models and Their X-Ray Reflection Signatures. I. Local Spectra, ApJ 546 (Jan., 2001) 406-418, [arXiv: astro-ph/0005597].

[8] R. R. Ross and A. C. Fabian, X-ray reflection in accreting stellar-mass black hole systems, MNRAS 381 (Nov., 2007) 1697-1701, [arXiv:0709. 0270].

[9] C. D'Angelo, D. Giannios, C. Dullemond, and H. Spruit, Soft X-ray components in the hard state of accreting black holes, AEA 488 (Sept., 2008) 441-450.

[10] H. C. Spruit, X-ray spectrum of a disk illuminated by ions, in LNP Vol. 487: Accretion Disks - New Aspects (E. Meyer-Hofmeister and H. Spruit, eds.), pp. 67-76, 1997.

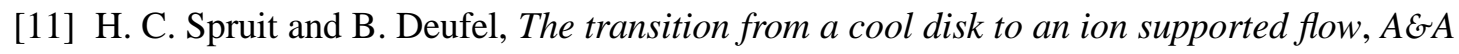
387 (June, 2002) 918-930, [astro-ph/0108497].

[12] C. P. Dullemond and H. C. Spruit, Evaporation of ion-irradiated disks, AEA 434 (May, 2005) 415-422, [astro-ph/0501463].

[13] B. Deufel and H. C. Spruit, Comptonization in an accretion disk illuminated by protons, $A \mathcal{E} A 362$ (Oct., 2000) 1-8.

[14] N. I. Shakura and R. A. Sunyaev, Black holes in binary systems. Observational appearance., AEA 24 (1973) 337-355.

[15] R. Narayan and I. Yi, Advection-dominated accretion: A self-similar solution, ApJ $\mathbf{4 2 8}$ (June, 1994) L13-L16, [astro-ph/9403052].

[16] R. R. Ross and A. C. Fabian, A comprehensive range of X-ray ionized-reflection models, MNRAS 358 (Mar., 2005) 211-216, [arXiv: astro-ph/0501116].

[17] T. Shimura and F. Takahara, On the spectral hardening factor of the X-ray emission from accretion disks in black hole candidates, ApJ 445 (June, 1995) 780-788. 